\title{
Gestionar castillos municipales en la provincia de Alicante (España): análisis de los casos de Castalla y Tibi
}

\section{Juan Antonio Mira Rico ${ }^{a}$, Daniel Martí i Pérez ${ }^{b}$ José Ramón Ortega Pérez}

${ }^{a}$ Universitat Oberta de Catalunya-ICOMOS España, Avenida República Argentina, 33, 50,03420 Castalla. jmirari@uoc.edu, ${ }^{\mathrm{b}}$ Arquitecto, Carrer Onil, 50, 03430 Onil.dmp@danielmarti.es y ${ }^{\mathrm{c} A R P A}$ Patrimonio, Avinguda del Rodalet, 23 A, 03690 Sant Vicent del Raspeig. arpapatrimonio@gmail.com.

\begin{abstract}
Resumen
Por el desarrollo histórico de España, como parte de al-Andalus, primero, y las coronas de Aragón y Castilla, después; la Comunidad Valenciana es un territorio privilegiado en cuanto a castillos se refiere. A modo de ejemplo, la provincia de Alicante, "solo" tiene, en este momento, 47 fortificaciones de propiedad municipal. Buena parte datan de la ocupación musulmana o surgieron como parte del sistema defensivo de la frontera sureste de la Corona de Aragón con la Corona de Castilla.

Por otro lado, muchas fortificaciones han sido objeto de estudios arqueológicos, arquitectónicos, históricos y de gestión. Este trabajo analiza la gestión de los castillos de Castalla y Tibi, situados al norte de la provincia de Alicante. Ambos, de origen musulmán e integrantes del sistema defensivo sureste de la Corona de Aragón, son municipales y presentan distintos grados de gestión. Castalla pasó del abandono, en los años ochenta del siglo XX, a la gestión profesional en la segunda década del siglo XXI. Por su parte, Tibi es una fortificación no gestionada en la que, ahora, se está empezando a revertir dicha situación mediante la elaboración de un plan director actualmente finalizado y pendiente de aprobación, por parte del Ayuntamiento de la localidad.
\end{abstract}

Palabras clave: Castalla, castillo, fortificación, gestión, provincia de Alicante, Tibi.

\begin{abstract}
The Valencian Community is a privileged territory in terms of castles due to the historical development of Spain, first as part of al-Andalus, and then as part of the crowns of Aragon and Castile. For instance, the province of Alicante has "only" 47 fortifications of municipal property. Many of them date from the Muslim occupation or emerged as part of the southeast border's defence of the Crown of Aragon against the Crown of Castile.

On the other hand, many of these fortifications have been studied from an archaeological, architectural, historical and management point of view. This paper analyses the management of Castalla Castle and Tibi Castle, located in the north of the province of Alicante. These municipal castles are Muslim and take part of the southeast border's defence of the Crown of Aragon. They also have different management degrees. Although Castalla Castle was abandoned during the eighties of the twentieth century, it became a professionally managed fortification in the second decade of the twenty-first century. For its part, Tibi Castle is an unmanaged fortification whose situation is beginning to be reversed thanks to the elaboration of a master plan-now completed-pending approval by the city council.
\end{abstract}

Keywords: Castalla, castle, fortification, management, province of Alicante, Tibi. 


\section{Alicante ¿tierra de castillos gestionados?}

Como se ha señalado en recientes trabajos, la provincia de Alicante (Comunidad Valenciana, España), presenta un gran patrimonio castellológico resultado de sus vicisitudes históricas como parte de al-Andalus, primero, y de la Corona de Aragón, después; con una gestión diversa en cuanto a nivel municipal se refiere (Fig. 1). En este sentido, y para el periodo comprendido entre los años 2003 y 2017, de los 42 castillos municipales estudiados en la provincia de Alicante, $12(28,57 \%)$ estaban gestionados de manera continuada, 18 (28,57\%) de manera puntual y $12(28,57 \%)$ se encontraban sin gestionar (Mira, 2017, p. 157, fig. 59) (Fig. 2).

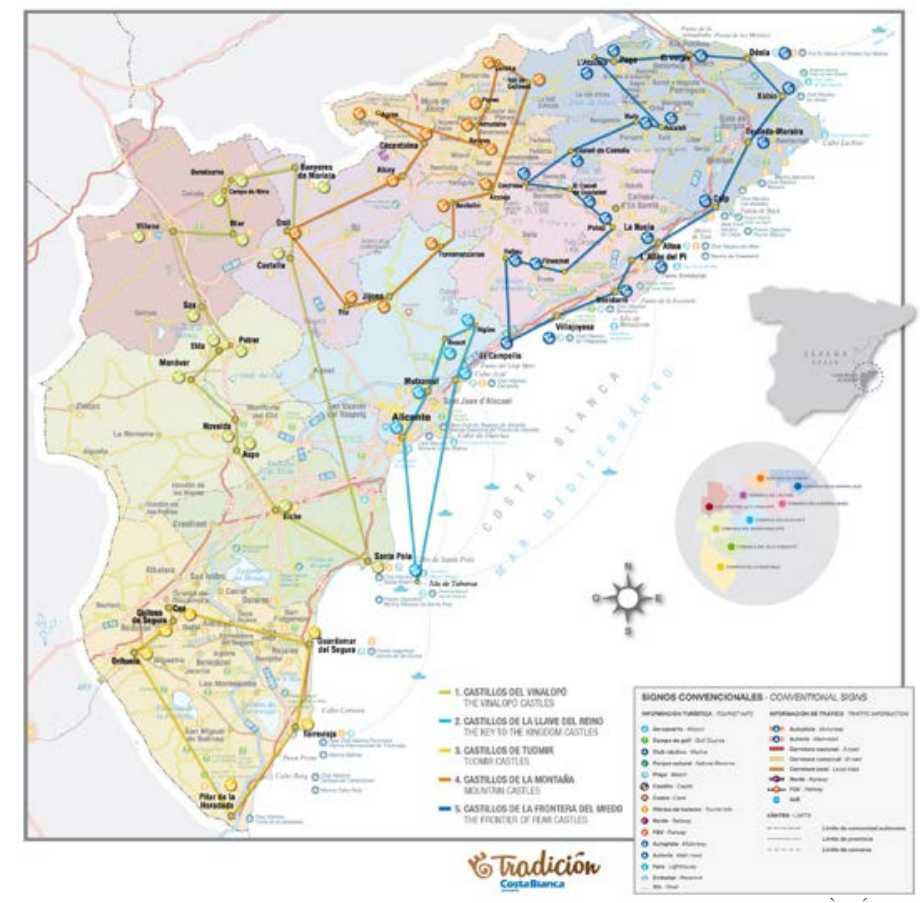

Fig. 1 Castillos y bienes de la arquitectura defensiva en la provincia de Alicante
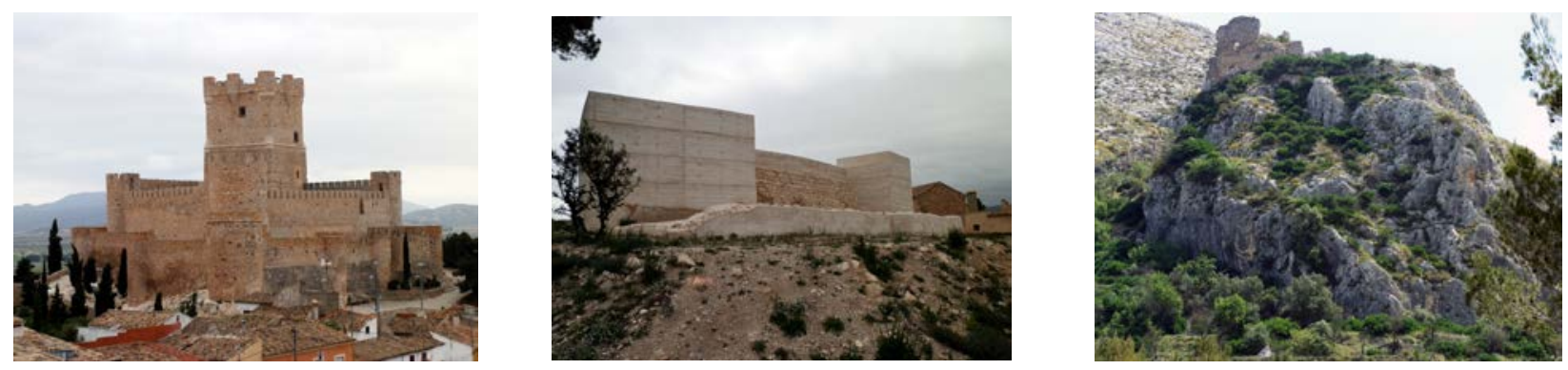

Autor: Juan Antonio Mira Rico

Fig. 2 Castillo de la Atalaya (Villena) (izda.), Castell d'Almizra (El Camp de Mirra), y Castell de Travadell (Millena) (dcha.). Ejemplos de fortificación gestionada (Atalaya), gestionada puntualmente (Almizra) y no gestionada (Travadell)

Dicha situación es consecuencia de la falta de recursos económicos y técnicos pero, también, de la ausencia de una planificación autonómica que articule y coordine las iniciativas que se desarrollan en las fortificaciones, similar al Plan Nacional de Arquitectura Defensiva, para el conjunto del estado español; el Plan Andaluz de Arquitectura Defensiva, para Andalucía; o la Scottish Castle Initiative, para Escocia (Mira, 2018). De esta manera, buena parte de las propuestas de gestión recaen en los propios propietarios de los castillos, ya sean públicos o privados. En el presente trabajo, centrado en las fortificaciones de Castalla y Tibi, de propiedad municipal y localizadas en las localidades de Castalla y Tibi, ubicadas en la comarca natural de la Foia de Castalla, al norte de la provincia de Alicante; se describirán las características fundamentales de la gestión desarrollada en la primera, entre los años 2009 y 2017, y las propuestas para iniciar la gestión de la segunda (pues hasta la fecha se encuentra sin gestionar). 


\section{Las fortificaciones de Castalla y Tibi: aspectos generales}
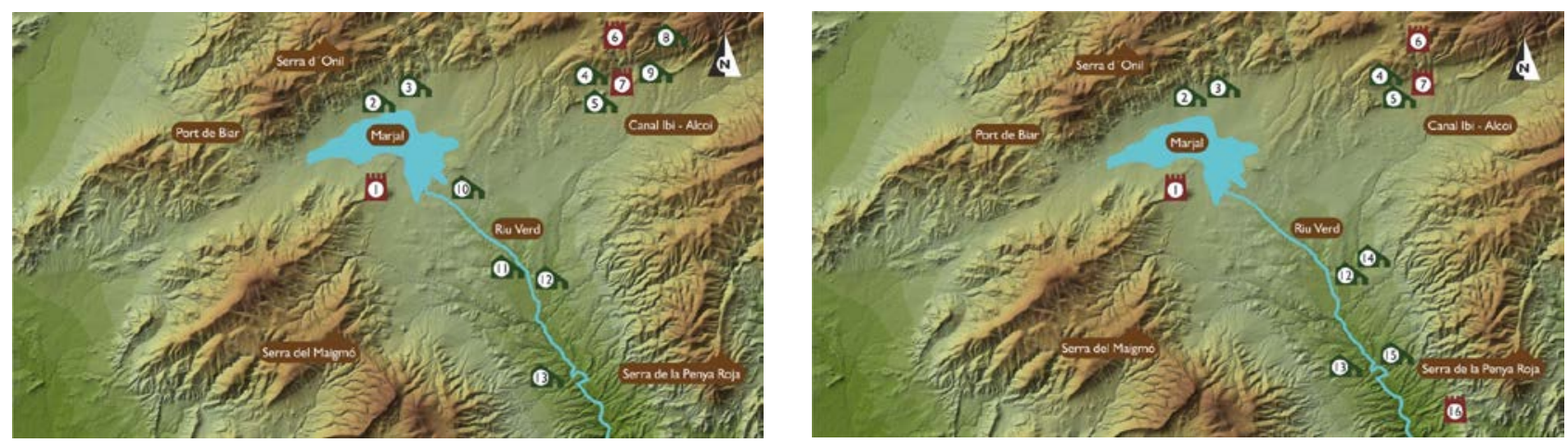

Autor: Juan Antonio Mira Rico, José Ramón Ortega Pérez y Atelier Proyectos

Fig. 3 Situación de los castillos de Castalla (1) y Tibi (16) en la comarca natural de Foia de Castalla durante los siglos XI-XV (periodo andalusí y cristiano bajomedieval)

El Castell de Castalla (Fig. 4) es una construcción andalusí, del siglo XI, que fue ocupada por los cristianos, de manera pacífica, en 1244, año en el que se convirtió en una pieza fundamental del sistema defensivo de la frontera sur del Reino de Valencia. Desde su edificación actuó como cabeza administrativa de la Foia de Castalla, articulando castillos menores, caso de las fortificaciones Vell (6), Vermell (7) y Tibi (16) y distintas alquerías Almarra (10), Cabanyes (12), Onil (2) o Sarganella (14) (Fig. 3) (Menéndez et al., 2010).

Consta de tres partes principales, el Palau ( $c a$. 1362-siglo XV), área residencial, con aljibe, despensas, cocina, cuerpo de guardia, etc.; el Pati d'Armes (siglos XI-XV), que albergaba las dependencias exteriores (aljibe, cobertizo-establo, cuerpo de guardia, murallas, etc.); y la Torre Grossa (ca. 1529), cuya función era residencial y militar.
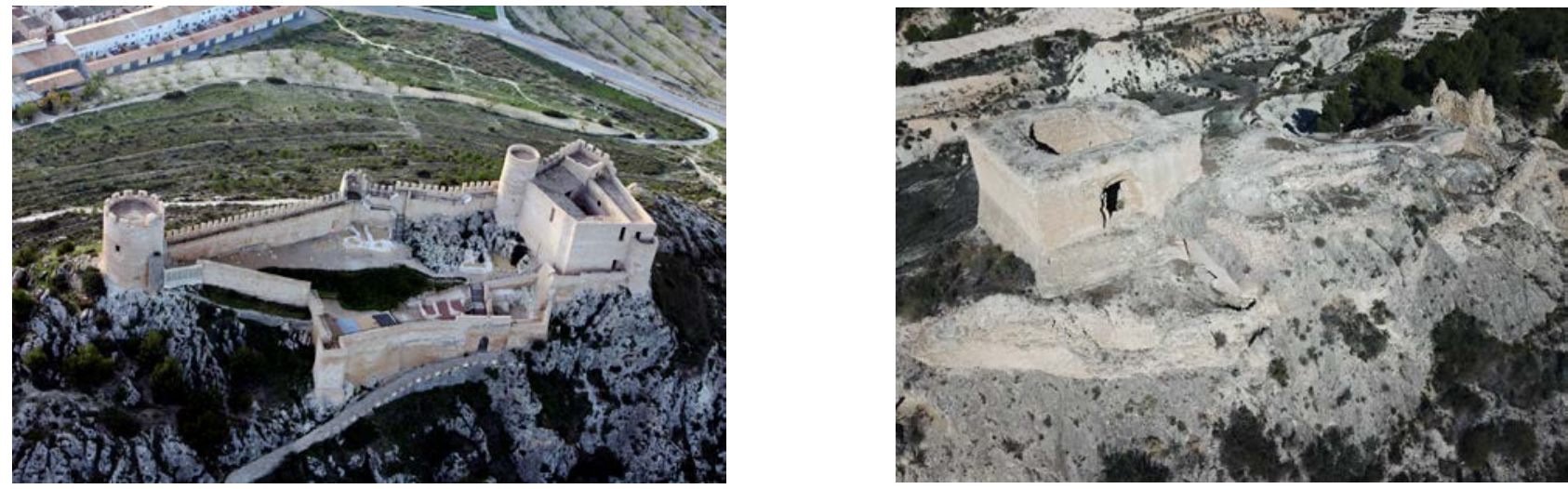

Autores: Andrés Ruiz Sánchez (Castell de Castalla) y Daniel Martí i Pérez y José Ramón Ortega Pérez (Castell de Tibi)

Fig. 4 Castell de Castalla y Castell de Tibi

Por su parte, el Castell de Tibi (Fig. 4), también, es una edificación andalusí, del siglo XII que, posiblemente, fue ocupado por los cristianos al mismo tiempo, o poco después, de la toma del Castell de Castalla. Del mismo sirvió como fortificación auxiliar, encargada de proteger el flanco sur de la Foia de Castalla, tanto en época andalusí como cristiana (Fig. 3) (Menéndez et al., 2010).

Consta de dos partes principales: el recinto superior y el recinto inferior, ambos con una cronología que iría, de manera hipotética, desde el siglo XII hasta el XVI. El primero de ellos alberga, entre otros, elementos andalusíes como una torre de tapial, dos aljibes y los tramos de muralla que siguen en pie, con una cronología del siglo XII-¿1244? También existen construcciones bajomedievales y modernas, con una cronología comprendida entre los siglos XIV y XVI, como la torre oriental, las modificaciones de la torre principal (puerta) y las estancias superiores. Por su parte, el recinto inferior concentraría un posible poblado andalusí, bajomedieval y moderno que iría desde el siglo XII hasta el XVI. 


\section{El Castell de Castalla y su gestión}

Hasta finales del siglo XX, el Castell de Castalla era una fortificación sin gestionar, situación que comenzó a cambiar en el año 1984, en el que empezó a gestionarse de manera puntual, a base a subvenciones, que permitieron llevar a cabo, entre otras intervenciones, la excavación arqueológica del Palau y el Pati d'Armes (1997-1999) y la intervención arquitectónica en todo el castillo (2003-2006) (Bevià, 2010; Mira y Ortega, 2015; Ortega y Esquembre, 2010). La elaboración del trabajo de investigación La gestión sostenible del patrimonio: propuestas de revalorización del patrimonio arqueológico del municipio de Castalla (Alicante) (Mira, 2005); permitió identificar una realidad patrimonial en el cerro del castillo, que iba más allá de la propia fortificación, estaba estrechamente relacionada entre sí y que había que tener en cuenta a la hora de administrar correctamente el castillo; pero de la que, paradójicamente, no se sabía nada porque la fortificación había acaparado, tradicionalmente, toda la atención de la sociedad -algo lógico, por otro lado, pues además de su importancia histórica y patrimonial, es el emblema de la localidad-. Dicha realidad fue denominada como Conjunt Patrimonial del Castell de Castalla, término que define la agrupación de bienes culturales materiales e inmateriales-, y naturales, con unos valores históricos, medioambientales y patrimoniales comunes, localizados y/o que se celebran en el promontorio del castillo (Mira et al., 2015, p. 381) (Fig. 5).

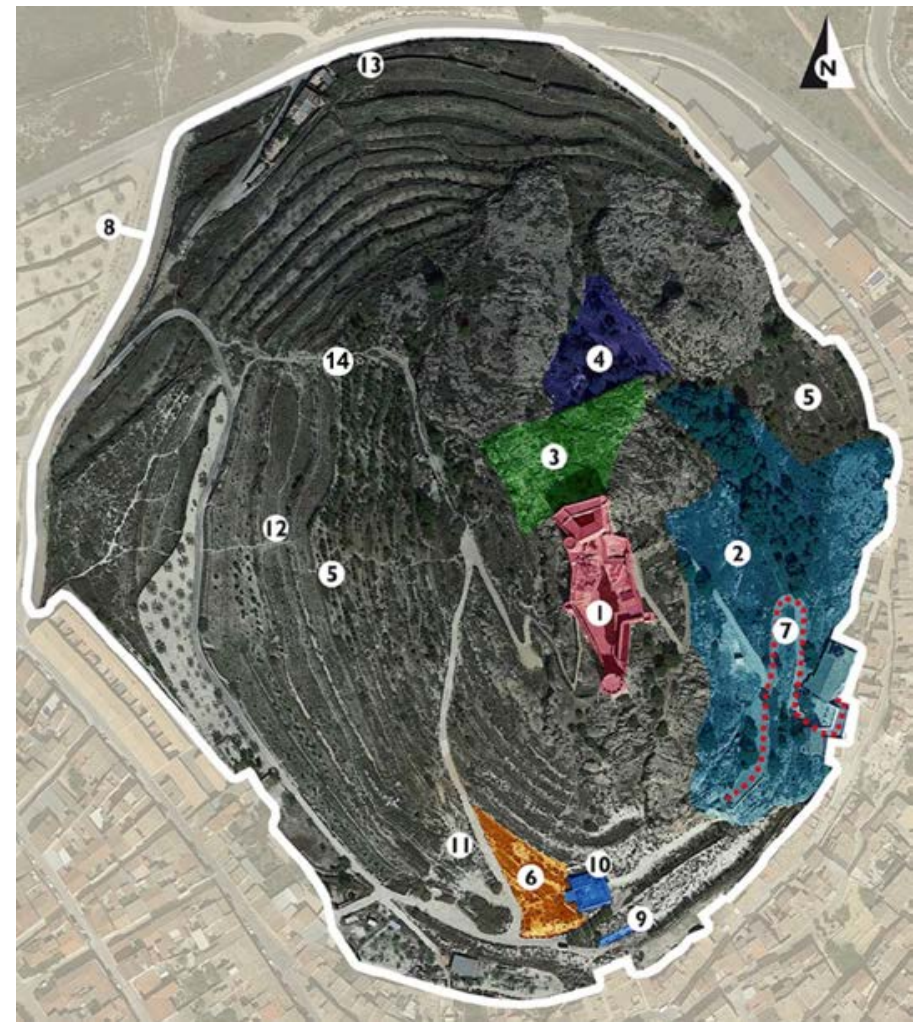

Autores: J.A. Mira Rico y Atelier Proyectos

Fig. 5 El Conjunt Patrimonial del Castell de Castalla en 2019. Patrimonio cultural material: 1-Castell de Castalla, 2-Vila medieval, 3-Posible albacara, 4-Posible necrópolis del Fossar Vell, 5-Márgenes y bancales de cultivo tradicionales, 6Yacimiento arqueológico del Dipòsit Vell, 7-Vías Crucis, 8-Escenarios bélicos de las batallas de Castalla, 9 y 10-Depósitos de agua, 11, 12 y 13-Refugios agrícolas y 14-Enterramientos de la Vessant Oest. Patrimonio cultural inmaterial: Fiestas de Moros y Cristianos y Semana Santa. Patrimonio natural: flora-224 especies pertenecientes a 59 familias diferentes de plantas y fauna-37 especies de vertebrados

A partir de aquí, el trabajo realizado en el seno del extinto Servei Municipal de Patrimoni Cultural de Castalla y de la Concejalía de Patrimonio Cultural del Ayuntamiento de Castalla, desde el año 2009, permitió profundizar y mejorar el conocimiento del conjunto patrimonial. En este sentido, conviene señalar que la superación del tándem patrimonio cultural igual a monumentos, aislados o incluyendo entorno y/o ambiente, y las obras de arte, a partir de la segunda mitad del siglo XX; permitió valorar, como patrimonio cultural, bienes no considerados como tal hasta la fecha. Se trata, de acuerdo con Juan Agudo Torrico (1999), de un cambio terminológico y de mentalidad, desde un modelo de 
gestión formado por el patrimonio histórico-artístico de carácter monumental y limitado a la cultura material; a otro integrado por el patrimonio cultural, focalizado en la cultura material e inmaterial y los bienes naturales antropizados (paisajes culturales) y caracterizado por ser coordinado, continuado, integral, local y público, como ya se ha puesto de manifiesto (Mira et al., 2015).

Este nuevo modelo, siguiendo los planteamientos de María Ángeles Querol Fernández (1995) y las experiencias desarrolladas en castillos del ámbito anglosajón como Dover Castle (Inglaterra), Castle Campbell (Escocia), Castle Hill National Historic Site (Canadá) o Castillo de San Marcos (Estados Unidos de América) (Fig. 6); puede considerarse, en cuanto a castillos se refiere, inédito en la provincia de Alicante. Por otro lado, se basa en la gestión integral de todos los bienes culturales y los naturales antropizados, sin importar su mayor o menor monumentalidad, valor o interés. Pues, sin dicha gestión siempre habría bienes abandonados e incluso marginados a favor de otros, actitud que, incluso, podría llegar a provocar su desaparición. Teniendo este tema presente, se diseñó y ejecutó, hasta 2017, el Proyecto de recuperación social del Conjunt Patrimonial del Castell de Castalla, dirigido por Juan Antonio Mira Rico, Màrius Bevià i Garcia y José Ramón Ortega Pérez. Este plan de gestión contó con la participación de más de veinte investigadores y profesionales especializados en el patrimonio cultural y natural; la financiación del Ayuntamiento de Castalla y la Generalitat Valenciana; y la colaboración estrecha de diversas concejalías del Ayuntamiento de Castalla Cultura, Medio Ambiente y Turismo-y de los centros educativos locales. El mismo se estructuró en una serie de pilares básicos -investigación, conservación, difusión, restauración y didáctica-, con el objetivo de recuperar todos los bienes culturales, inmateriales y materiales, y naturales del conjunto patrimonial.
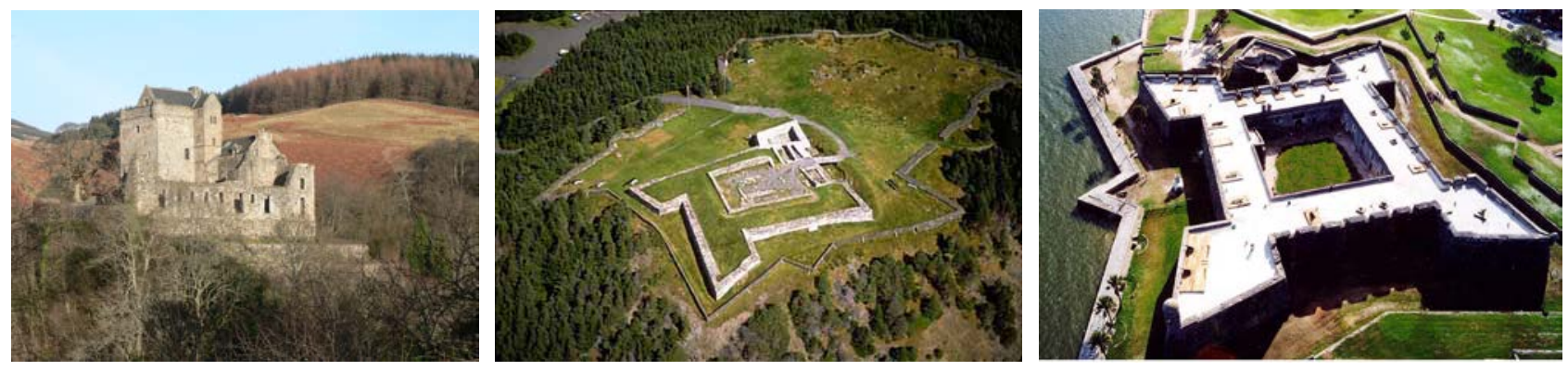

Fuente: https://en.wikipedia.org/wiki/Castle_Campbell, https://www.pc.gc.ca/apps/dfhd/page_nhs_eng.aspx?id=230 y https://es.wikipedia.org/wiki/Castillo_de_San_Marcos_(Florida)

Fig. 6 Castle Campbell (Escocia) (izda.), Castle Hill National Historic Site (Canadá) y Castillo de San Marcos (EUA) (dcha.)

\section{El Castell de Tibi y su gestión}

En el Castell de Tibi, un castillo no gestionado (Mira, 2017, pp. 104-112), se están dando los primeros pasos del largo proceso que supone la puesta en valor y recuperación de la fortificación -que, también, reúne valores para ser considerado como un conjunto patrimonial-; para su posterior gestión de una forma sostenible y sostenida en el tiempo. Se puede considerar que hasta la fecha, las actuaciones llevadas a cabo no han supuesto ningún avance en este sentido o no han tenido continuidad con lo que las inversiones y los esfuerzos han sido baldíos. Es el caso, por ejemplo, de las excavaciones arqueológicas, fruto de un convenio entre el Ayuntamiento de Tibi y el antiguo INEM, realizadas por el equipo dirigido por el entonces arqueólogo Francisco Franco Sánchez -ahora catedrático de Estudios Árabes e Islámicos en la Facultad de Filosofía y Letras de la Universidad de Alicante-; en el recinto superior y en la torre principal del castillo (1985). De dicha intervención únicamente nos han llegado dos breves trabajos incluidos en la publicación Memòries arqueològiques de la Comunitat Valenciana 1984-1985 (Franco, 1988), además, claro está, del material arqueológico exhumado que se encuentra depositado en el Museo Arqueológico Provincial de AlicanteMARQ.

Hasta la redacción del Plan Director del Castillo Almohade de Tibi (Martí, 2019), que se empezó a elaborar en el último trimestre de 2018 y, actualmente, se encuentra finalizado y en fase de aprobación definitiva; no se llevó a cabo otra actuación destacada en la fortificación. La iniciativa para elaborar dicho documento surgió de la voluntad del Ayuntamiento de Tibi por recuperar y poner en valor el castillo. Para ello, y al no disponer de los medios suficientes 
para acometer la empresa por si mismo, solicitó la participación de la Diputación de Alicante que, en estos momentos, cuenta con una línea de trabajo de planes directores para fortificaciones situadas en municipios de pequeño y mediano tamaño caso de Benifallim, Perputxent y Tàrbena (Mira, 2018, p. 1307).

El objetivo general de dicho documento es convertir al castillo en un conjunto patrimonial plenamente valorizado y correctamente gestionado, en la línea del Conjunt Patrimonial del Castell de Castalla y de los ejemplos foráneos ya expuestos. Para ello, se quieren sentar las bases necesarias para que la posterior redacción de proyectos (puntuales o de carácter más global y general) y la realización de cualquier actuación encaminada a la puesta en valor de la fortificación dispongan de un marco de actuación que permita estructurarlas. De este modo, las instituciones, organismos y equipos técnicos involucrados en el proceso de valorización, primero, y de gestión, después, del Conjunt Patrimonial del Castell de Tibi; dispondrán de un guion de actuación, a cuya conclusión se habrá podido llevar a cabo una puesta en valor del conjunto patrimonial satisfactoria con una correcta gestión de los recursos a ella destinados (Martí, 2019).

Tras el estudio de la información existente y de los restos arquitectónicos encontrados, se identificaron una serie de espacios que han sido objeto de un exhaustivo análisis que ha permitido organizarlos en áreas de actuación que servirán de base para la elaboración de los diferentes planes de intervención (Fig. 7).

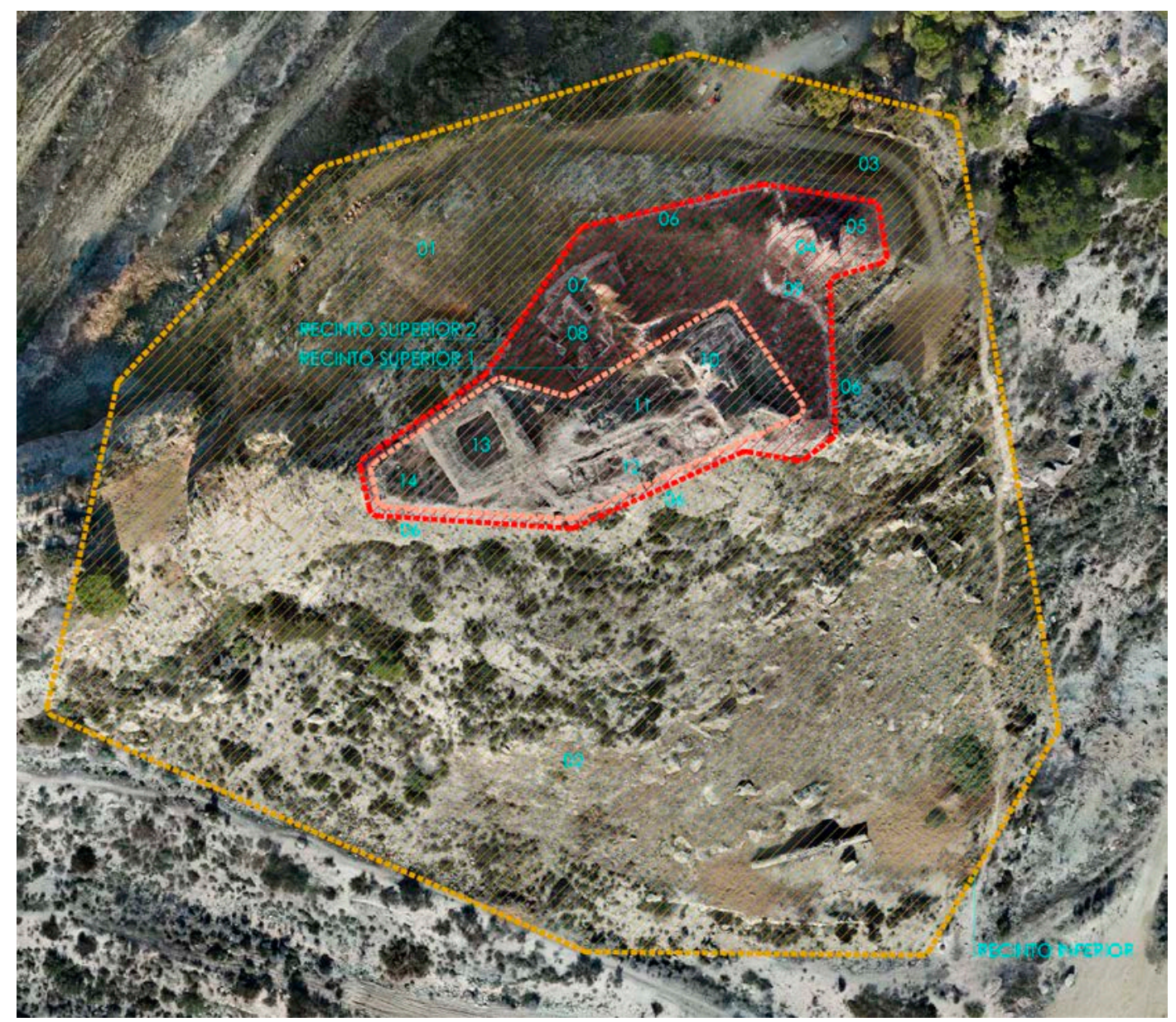

Autores: Daniel Martí i Pérez y José Ramón Ortega Pérez

Fig. 7 Elementos del Castell de Tibi: Recinto inferior: 1-Ladera norte, 2-Ladera sur, 3-Foso; Recinto superior 2: 4-Torre Oriental, 5-Plataforma Oriental, 6-Murallas, 7-Aljibe inferior, 8-Estancias septentrionales, 9-Acceso; Recinto superior 1: 10Aljibe superior, 11-Estancias centrales, 12-Estancias meridionales, 13-Torre principal y 14-Plataforma occidental

El estudio de estos espacios y de sus necesidades atendiendo a los estados de conservación que presenten serán la base para la propuesta de las diferentes intervenciones que el plan director prevé llevar a cabo en el plazo de unos 8 años. Para ello se establece un cronograma de etapas y fases de actuación (Fig. 8) que, según los criterios de mínima intervención, reversibilidad de las actuaciones y fácil identificación de los rasgos originales, procurará que las 
actuaciones sean de una escala y presupuesto asumibles para un municipio como Tibi. Dichas intervenciones están concebidas para que, tras su finalización, puedan ser abiertas al público y ayuden a conferir un perfil reconocible del castillo para los visitantes y vecinos del municipio. Este esfuerzo tendrá como resultado final la puesta en valor del conjunto patrimonial y el establecimiento de un sistema de gestión que garantice su sostenibilidad (Martí, 2019).

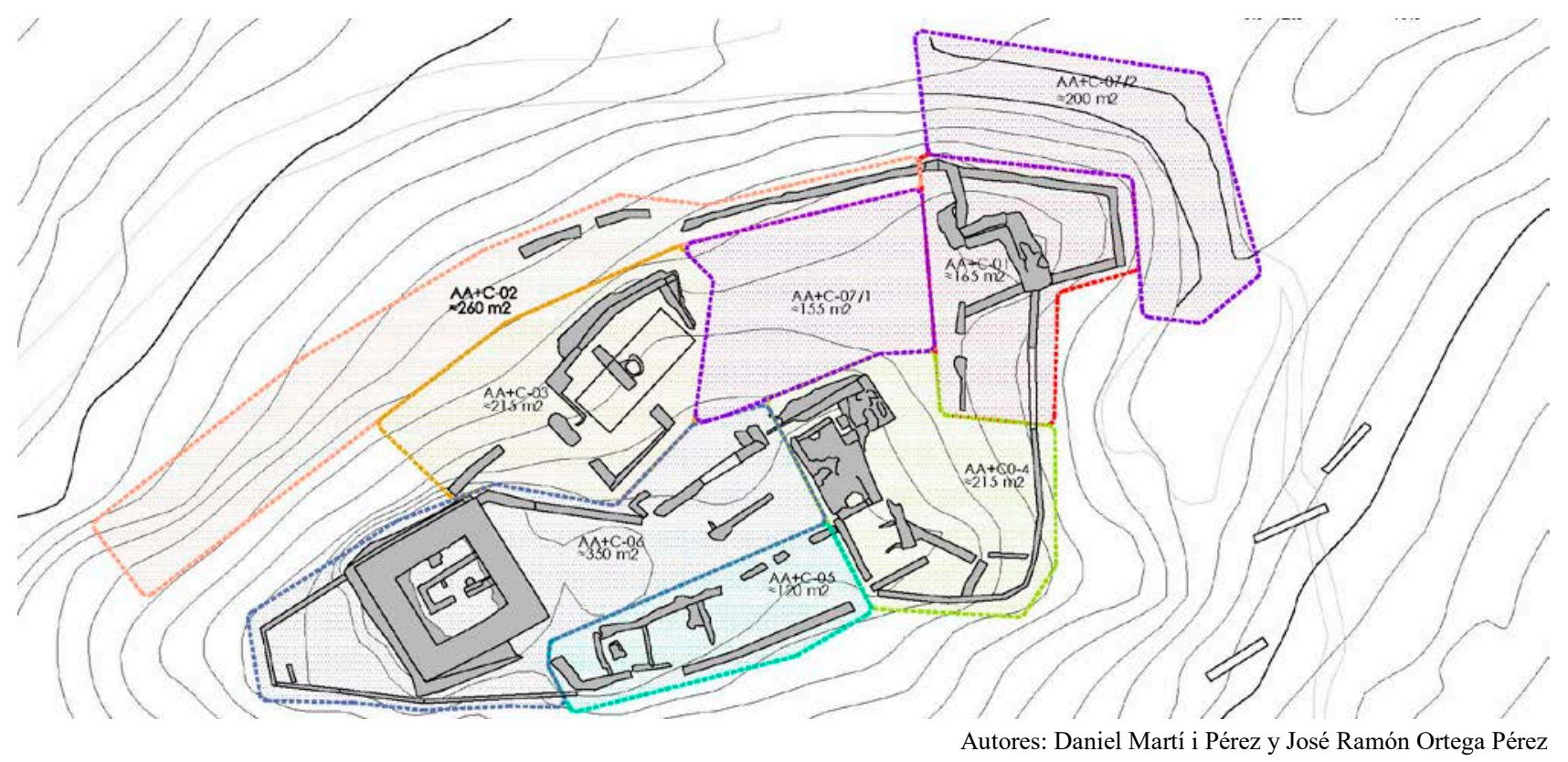

Fig. 8 Plano de Áreas de Actuación + Consolidación

\section{Conclusiones}

Los castillos de la provincia de Alicante, en cuanto a los de propiedad municipal se refiere, no presentaban durante el periodo comprendido entre los años 2003 y 2013 y a nivel de gestión, una situación óptima. Como se ha señalado, de las 42 fortificaciones estudiadas, 12 estaban gestionadas de manera continuada, 18 lo estaban puntualmente y 12 estaban sin gestionar (Mira, 2017, p. 157, fig. 59). En estos momentos, la situación tiene visos de mejorar, pues la Diputación de Alicante está impulsando la realización de una serie de planes directores en distintas fortificaciones que, en 2013, se encontraban sin gestionar. Es el caso, por ejemplo, de Benifallim, Tàrbena y Tibi (Mira, 2017, pp. 167 y 168, tabla 69; 2018, p. 1307). A los esfuerzos de la Diputación de Alicante, hay que sumar los de la Generalitat Valenciana. La administración autonómica ha puesto en marcha, gracias a los Fondos FEDER para la Comunitat Valenciana 2014-2020, destinados a las entidades locales, un maná de dinero para la protección, conservación, recuperación y puesta en valor del patrimonio cultural valenciano. En el caso de la provincia de Alicante, de las 36 actuaciones previstas, casi la mitad, 14, tienen lugar en castillos, caso del Aljau (Aspe), Ambra (Pego), Benidorm, Biar, Busot, Callosa de Segura, Castalla, Cocentaina, Dénia, Guardamar del Segura, Ocaive (Pedreguer), Orba, San Fernando (Alicante) y Torre Grossa (Xixona). Y de ellas, 11 se realizan en fortificaciones estudiadas que, hasta 2013, presentaban distintos niveles de gestión: de gestión continuada son las fortificaciones del Aljau (Aspe), Biar, Castalla, Cocentaina y Dénia; de gestión puntual son los castillos de Ambra, (Pego), Busot, Callosa de Segura, Guardamar del Segura, Torre Grossa (Xixona); y sin gestión se encontraba el Castell d'Ocaive (Pedreguer) (Mira, 2017, pp. 167 y 168, tabla 69). Queda por ver si dicha inversión permite las fortificaciones gestionadas puntualmente o que se encontraban sin gestionar, pasar a ser gestionadas habitualmente.

En dicho contexto, el Conjunt Patrimonial del Castell de Castalla, en general, y la fortificación, en particular, formaron, entre los años 2009 y 2017, parte de un proyecto basado en la gestión integral del patrimonio, cultural y natural (investigación, conservación, restauración, didáctica y difusión); cuyo epicentro era el contexto local. Se trató de una iniciativa novedosa por su promotor, un ayuntamiento de mediano tamaño, y por implementar un modelo de gestión global, cuando, hasta la fecha, toda la atención recaía en el castillo; encaminada a convertir el conjunto patrimonial en 
el primer parque cultural de estas características de la Comunidad Valenciana, pues, como se demostró, tenía las condiciones para ello (Mira, 2016). Si bien es cierto que no fue la única fortificación de la provincia de Alicante que apostó por dicha línea, pues Sax también lo hizo, aunque la gestión todavía sigue focalizada en el castillo. Volviendo al conjunto patrimonial, por un lado su transformación en parque cultural es algo que, todavía, no se ha conseguido y, por otro, en los últimos años (2018-presente), se aprecia un cambio de tendencia. En este sentido se ha pasado de una gestión continuada a una gestión puntual basada en subvenciones, pues todas las actuaciones realizadas en dicho periodo lo han sido porque había subvenciones y no por la aplicación, sistemática, del plan de gestión.

Por su parte, Tibi está dando los primeros pasos para gestionar su fortificación que, por sus valores patrimoniales culturales y naturales, también puede ser considerada como un conjunto patrimonial. Ahora se encuentra en el primer estadio que ha de sentar las bases de futuras actuaciones para que, siguiendo la senda marcada por el Conjunt Patrimonial del Castell de Castalla, con el que, por razones obvias, habría que establecer sinergias, al igual que con otras fortificaciones asentadas en municipios de pequeño y mediano tamaño; pase de un estado de abandono a estar gestionado de manera continuada para aprovechar su potencial cultural y turístico en el contexto de la provincia de Alicante.

\section{Referencias}

Agudo Torrico, J. (1999). Cultura, patrimonio etnológico e identidad. En PH: Boletín del Instituto Andaluz del Patrimonio Histórico $7(29), 36-45$.

Bevià i Garcia, M. (2010). La intervención arquitectónica en el Castell de Castalla (2003-2006). En J. L. Menéndez Fueyo, M. Bevià i Garcia, J. A. Mira Rico y J. R. Ortega Pérez (Eds.), El Castell de Castalla. Arqueología, arquitectura e historia de una fortificación de frontera (pp. 19-30). Alicante: MARQ.

Franco Sánchez, F. (1988). El Castell de Tibi, Alcoià. En Memòries arqueològiques a la Comunitat Valenciana 1984-1985 (pp. 2833). Valencia: Generalitat Valenciana.

Martí i Pérez, D. (2019). El Plan Director del Castillo Almohade de Tibi. Trabajo inédito. Onil: Arquitectura DMP.

Menéndez Fueyo, J. L., Bevià i Garcia, M., Mira Rico, J. A., y Ortega Pérez, J. R. (2010). El Castell de Castalla. Arqueología, arquitectura e historia de una fortificación de frontera. Alicante: MARQ.

Mira Rico, J. A. (2018). Por un plan autonómico para la gestión de los castillos en la Comunidad Valenciana (España). En A. Marotta y R. Spallone (Eds.), Proceedings of the International Conference on Modern Age Fortifications of the Mediterranean Coast FORTMED 2018, International Conference on Modern Age Fortifications of the Mediterranean Coast FORTMED 2018. Turín: Politecnico di Torino.

Mira Rico, J. A. (2017). Management Analysis of Municipal Castles in the Province of Alicante (Spain). Oxford: BAR Publishing.

Mira Rico, J. A. (2016). Castles or cultural and natural landscapes? A new approach to the management of fortifications in the south of the Valencian Community (Spain). Examples of Castalla and Sax (Alicante). En P. Kolodziejczyk y B. Kwiatkowska-Kopka (Eds.), Cracow Landscape monographs: Cracovia: Jagiellonian University in Kraków y Cracow University of Technology.

Mira Rico, J. A. (2005). La gestión sostenible del patrimonio: propuestas de revalorización del patrimonio arqueológico del municipio de Castalla (Alicante). Trabajo de Licenciatura. Sant Vicent del Raspeig: Universidad de Alicante.

Mira Rico, J. A. y Ortega Pérez, J. R. (2015). Castalla Castle. Architecture and restoration in the 21st century in Alicante. En R. Amoêda, S. Lira y C. Pinheiro (Eds.), REHAB 2015. Proceedings of the $2^{\text {nd }}$ International Conference on Preservation, Maintenance and Rehabilitation of Historical Buildings and Structures, $2^{\text {nd }}$ International Conference on Preservation, Maintenance and Rehabilitation of Historical Buildings and Structures. Barcelos: Green Lines Institute.

Mira Rico, J. A., Bevià i Garcia, M., y Ortega Pérez, J. R. (2015). Del Castell de Castalla al Conjunt Patrimonial del Castell de Castalla: un nuevo enfoque en la gestión del patrimonio cultural valenciano. En P. Rodríguez-Navarro (Ed.), Proceedings of the International Conference on Modern Age Fortifications of the Western Mediterranean Coast Defensive Architecture of the Mediterranean XV to XVIII Centurie. Valencia: Universitat Politècnica de València.

Ortega Pérez, J. R. y Esquembre Bebia, M. A. (2010). Intervención arqueológica en el recinto fortificado del Castell de Castalla. En J. L. Menéndez Fueyo, M. Bevià i Garcia, J. A. Mira Rico y J. R. Ortega Pérez (Eds), El Castell de Castalla. Arqueología, arquitectura e historia de una fortificación de frontera (pp. 61-106). Alicante: MARQ. 